\title{
Eye-contact and individual looking: The role of chance
}

\section{H. Wagner, A. H. Clarke and J. H. Ellgring}

"If chance robots are indulging in directed gaze, where looking towards is "on" and looking away is "off", the amount of mutual "on" which occurs can be calculated probabilistically' (Strongman \& Champness, 1968) as the product of the individual looking levels (ILLs). Implicit to the "gazing robots' model is that the gaze behaviour of the participants is mutually independent. It follows that the accuracy of the estimated value of eye-contact should decrease when mutual dependence does in fact exist. In accordance with this thesis, various authors have adopted the deviation between empirically measured and estimated amount of eye-contact as an indicator of the mutual influence of the ILLs (Argyle \& Dean, 1965; Rubin, 1970; Argyle \& Ingham, 1972; Rutter et al., 1977).

Whereas earlier reports test the assumption that the relationship between individual gaze is not random, and test for deviations between empirical and estimated values, Rutter et al. (1977) assume the random hypothesis, their examination of the correlation between empirical and estimated EC yielding values of the order of 'at least 0.97 '. Nevertheless, some caution is required when interpreting this high correlation as adequate proof of randomness.

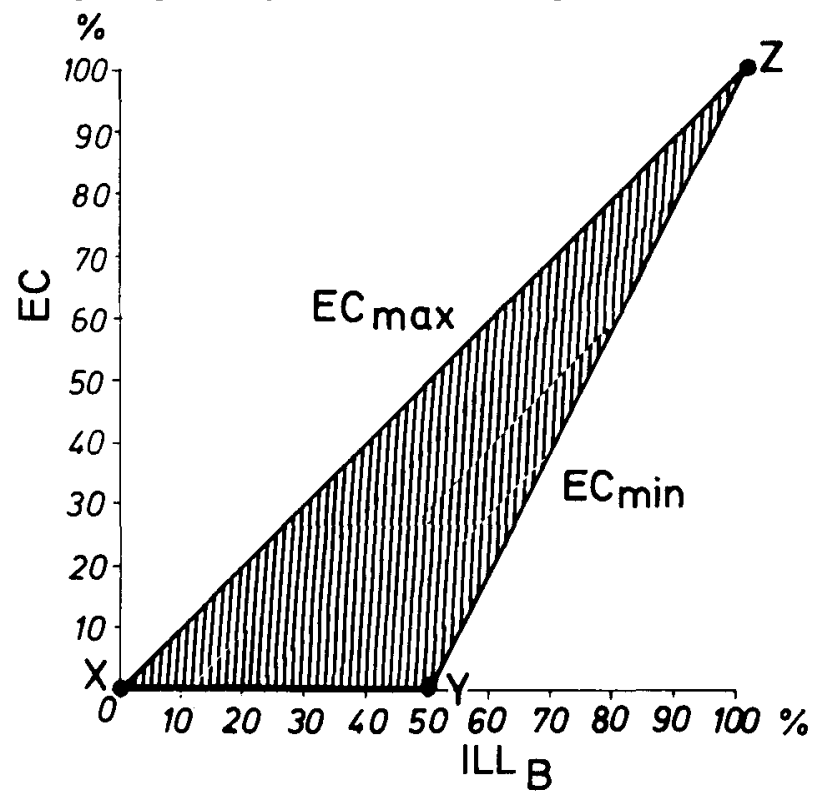

Figure 1. Relationship between eye-contact and individual looking levels.

It can be demonstrated that a restrictive mathematical relationship exists between EC and ILLs. This is illustrated in Fig. 1. The maximum possible duration of mutual gaze (ECmax) is equal to the ILL of that participant (B) who looks less, i.e.

$$
\mathrm{EC} \max =\mathrm{ILL} B \%
$$

(corresponding to ' $\mathrm{XZ}$ ' in Fig. 1).

The lower limit for mutual gaze (ECmin, resp. ' $\mathrm{XYZ}$ ' in Fig. 1) can be calculated from the ILLs of both participants, i.e.

$$
\mathrm{ECmin}=(\text { ILL A + ILL B - 100)\%, }
$$

so that ECmin can be equal to 0 , if ILL $A+$ ILL $B \leqslant 100 \%$. Thus, given any value of ILL B, $\mathrm{ECmin} \leqslant \mathrm{EC} \leqslant \mathrm{ECmax}$; the range of possible values is defined. For example,

for ILL $A=70 \%$ and ILL $B=60 \%, 30 \% \leqslant \mathrm{EC} \leqslant 60 \%$, or

for ILL $A=80 \%$ and ILL $B=20 \%, 0 \% \leqslant E C \leqslant 20 \%$, or

for ILL $\mathrm{A}=50 \%$ and ILL $\mathrm{B}=50 \%, 0 \% \leqslant \mathrm{EC} \leqslant 50 \%$. 
In fact, all possible values for mutual gaze (EC) fall within the limits of the area shown in Fig. 1. As can be seen, the range decreases with increasing ILL so that a higher correlation can be expected between observed and estimated values. This substantial limitation to the possible range of variation of EC amount offers some explanation of the high correlation between estimated and empirical values.

Further, not all points on the enclosed surface are likely to be realized. Considering the third example above, all values between 0 per cent and 50 per cent are possible, but the extremes would presume exceptional coordination in mutual looking behaviour. Thus, the most likely EC levels, in terms of psychological least effort, should tend to lie around the middle line of the triangle. According to the principle of least effort, the easiest way to attain the intended level of EC is the regulation of the ILL: i.e. the individual maintains a high enough ILL to produce the desired level of EC.

This can be understood as tonic regulation of EC level.

As can be inferred from the reports of Rubin (1970), Argyle \& Ingham (1972) and Russo (1975), the role of chance is further narrowed by the selective adjustment of the duration of each eyecontact event according to the momentary functional requirements. When random eye-contact occurs, it may be extended (cf. Rubin's romantic lovers or Russo's schoolfriends) or cut short (cf. Argyle \& Ingham's strangers under intimacy pressure). Accordingly, participants adjust their ILLs to their individual cognitive, emotional and communicative requirements in the given situation. This may be understood as phasic regulation of eye-contact.

The distinction between tonic and phasic aspects helps to resolve the apparent contradiction that eye-contact is a socially important signal, but can be modelled as the chance product of individual looking.

This requires some qualification of the suggestion by Rutter et al. (1977): 'While we have assumed that the level of eye-contact is determined by individual looking, the reverse may actually be the case.' In fact, both may be true; the level of EC may be determined-statistically - by calculation from the ILLs, whereas-psychologically-the ILLs are influenced by the predisposition of the individuals to enter into ECs.

\section{Acknowledgements}

This paper was written in connection with a project on human communication supported by the Deutsche Forschungsgemeinschaft No. E1 67/2.

\section{References}

Argyle, M. \& Dean, J. (1965). Eye contact, distance, and affiliation. Sociometry, 28, 289-304.

Argyle, M. \& Ingham, R. (1972). Gaze, mutual gaze, and proximity. Semiotica, 6, 32-49.

Rubin, A. (1970). Measurement of romantic love. Journal of Personality and Social Psychology, 16, 265-273.

Russo, N. F. (1975). Eye contact, interpersonal distance, and the equilibrium theory. Journal of Personality and Social Psychology, 31, 497-502.

Rutter, D. R., Stephenson, G. M., Lazzerini, A. J., Ayling, K. \& White, P. A. (1977). Eye-contact: A chance product of individual looking? British Journal of Social and Clinical Psychology, 16, 191-192.

Strongman, K. T. \& Champness, B. G. (1968). Dominance hierarchies and conflict in eye-contact. Acta Psychologica. 28, 376-386.

Received 3 November 1981; revised version received 17 March 1982

Requests for reprints should be addressed to H. Wagner, MPI-Psychiatry, Kraepelinstrasse 10, 8000 Munich 40, W.Germany.

A. H. Clarke and J. H. Ellgring are also at the MPI-Psychiatry. 